\title{
THE EFFECT OF MECHANICAL REPERFUSION OUTCOME FOLLOWING AN OCCLUSIVE STROKE ON THE REGIONAL EXPRESSION OF MATRIX METALLOPROTEINASE-9 IN RAT BRAIN
}

Andrew Douglas ${ }^{1,2}$, Jennifer Shearer², Abhay Pandit', Karen Doyle ${ }^{1,2}$

${ }^{1}$ Centre for Research in Medical Devices, National University of Ireland, Galway, ${ }^{2}$ Physiology Department, National University of Ireland, Galway.

\section{BACKGROUND}

$>15$ million people suffer from a stroke each year; over $80 \%$ of strokes are caused by occlusion.

$>$ Early reperfusion can potentially minimise the degree of brain damage in a stroke caused by occlusion.

$>$ However, reperfusion also can result in serious complications due to abnormal permeability of the blood brain barrier.

$>$ Increased expression of matrix metalloproteinase-9 (MMP9) in brain tissue is a useful marker of blood brain barrier dysfunction ${ }^{1}$.

\section{AIMS}

$>$ To study the effect of different mechanical reperfusion outcomes following occlusive stroke on a marker of blood brain barrier dysfunction - matrix metalloproteinase-9 (MMP-9)

\section{METHODOLOGY}

> Male Sprague Dawley rats underwent 2 hour middle cerebral artery occlusion (MCAO) ( $n=6-14$ per group).

$>$ Following occlusion, different reperfusion strategies were employed for a further 2 hours; either full, partial or no reperfusion, simulating cerebral blood flow (CBF) restoration that may occur during thrombectomy. In shamoperated animals, filament insertion was omitted.

> Blood pressure, heart rate, blood gases, body temperature and blood glucose were closely monitored and controlled.

$>$ Cerebral blood flow (CBF) was measured using laser Doppler flowmetry.

- Serial coronal brain slices $(2 \mathrm{~mm}$ thick) were incubated in $1 \% \mathrm{w} / \mathrm{v} 2,3,5$-triphenyltetrazolium chloride (TTC) solution at $37^{\circ} \mathrm{C}$ for 20 minutes to calculate the percentage hemispheric lesion volume (\% HLV) and \% oedema.

$>$ Brain slices were subsequently dissected and the expression of MMP-9 in the frontal cortex, striatum, anterior temporal cortex, posterior temporal cortex and hippocampus was assessed by ELISA (DuoSet, R\&D Systems).

\section{RESULTS}

Blood pressure, heart rate, blood gases, body temperature and blood glucose were all stable and within normal physiological limits throughout.

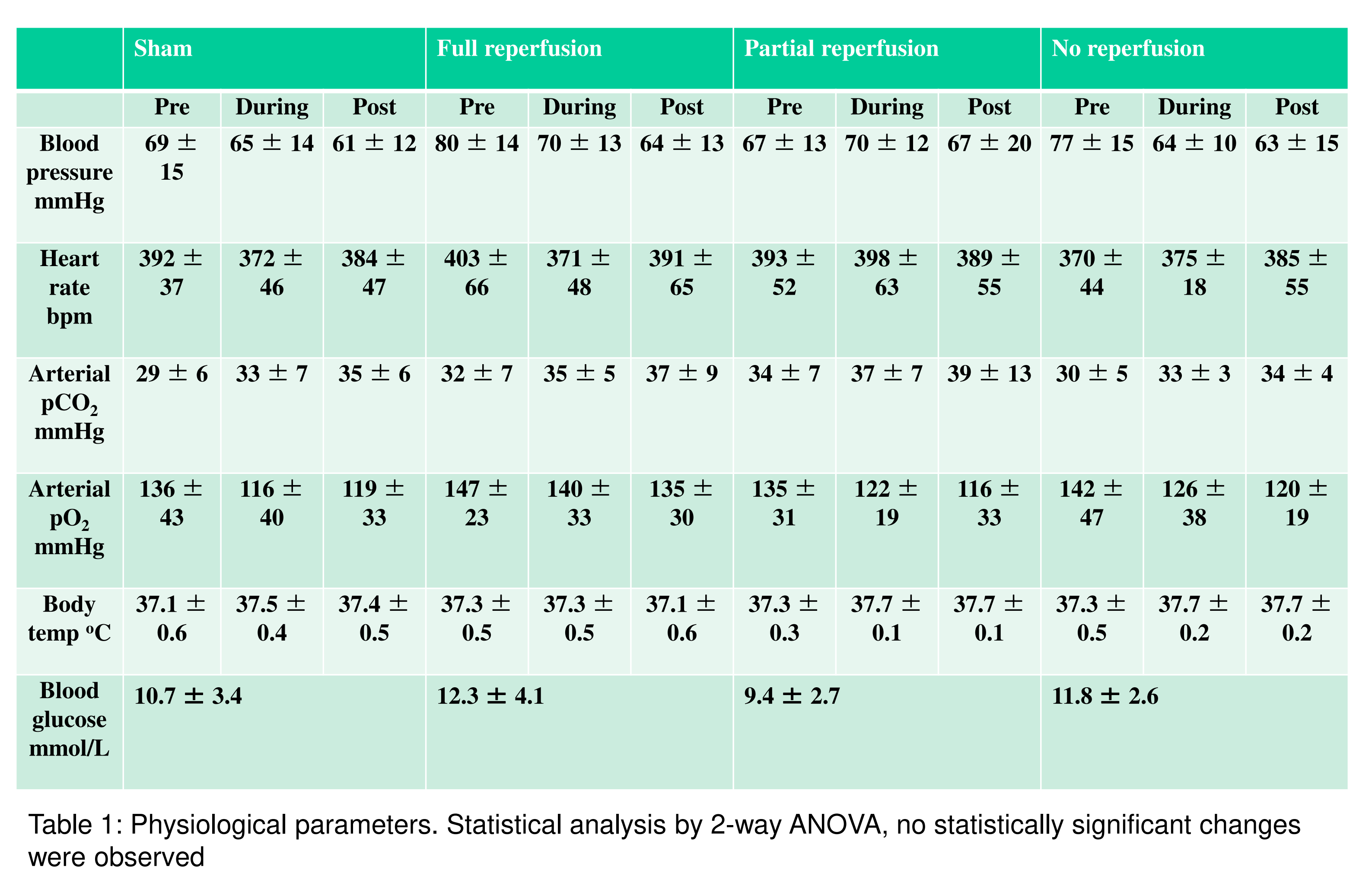

\section{REFERENCES}

1. Heo, J. H., et al. (1999) Matrix metalloproteinases increase very early during experimental focal cerebral ischemia Journal of Cerebral Blood Flow \& Metabolism 19 (6): 624-633

\section{RESULTS}

$>\mathrm{MCAO}$ caused a drop in CBF to $50 \%$ of pre-occlusion level $(F(3,37)=10.57 ; p<0.001)$ (Fig 1). Reperfusion approach had a significant effect on $\operatorname{CBF}(F(2,27)=5.12 ; p<0.05) ; 78 \%, 53 \%$ and $43 \%$ of sham levels for full, partial and no reperfusion groups respectively (Fig 2 ).
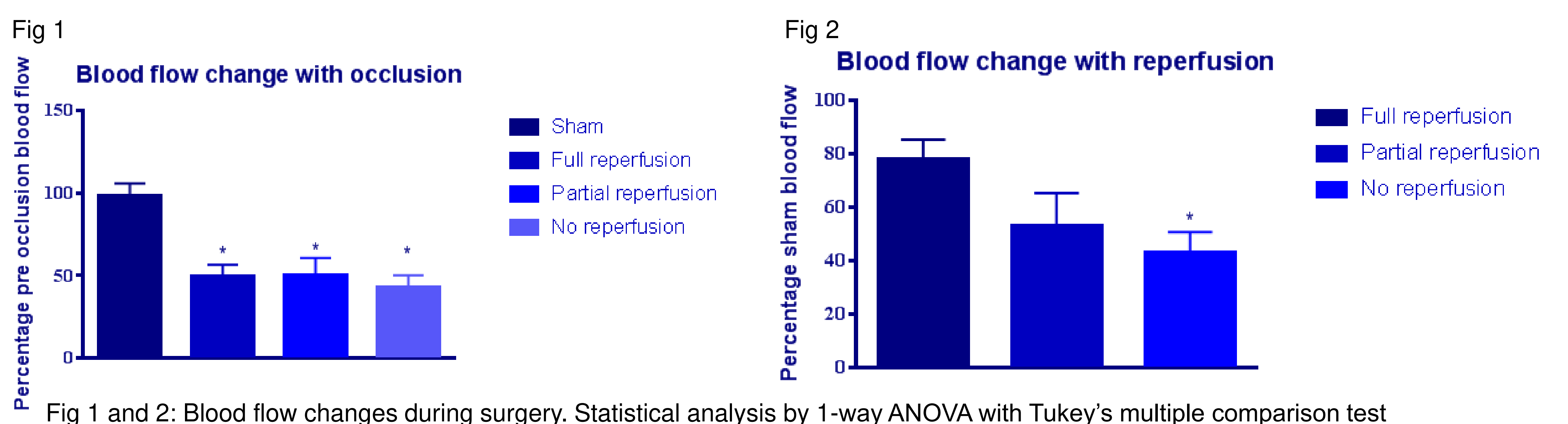

$>$ Occlusion resulted in significant infarction $(F(3,26)=3.03 ; p<0.05)$, most prominent in nonreperfused animals (Fig 3), and also significant oedema $(F(3,26)=5.49 ; p<0.01)$ (Fig 4$)$.
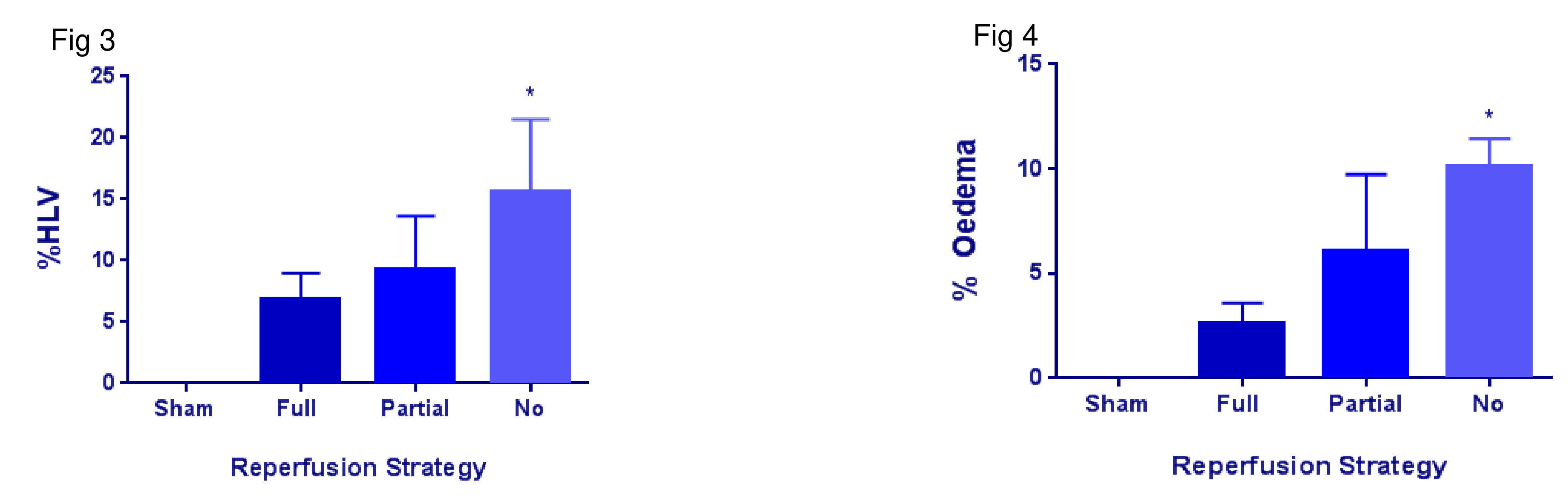

Fig 3 and 4:\% HLV and oedema. Statistical analysis by 1-way ANOVA with Tukey's multiple comparison test

$>$ Occlusion resulted in increased MMP-9 expression in the lesioned hemisphere in the frontal cortex $(F(1,52)=15.51 ; p<0.001)$ (Fig 5$)$, striatum $(F(1,52)=39.98 ; p<0.001)$ (Fig 6$)$ anterior temporal cortex $(F(1,52)=63.85 ; p<0.001)$ (Fig 7) and posterior temporal cortex $(F(1,52)=13.34 ; p<0.001)$ (Fig 8), but not the hippocampus $(F(1,52)=2.00 ; n s)$ (Fig 9$)$.

$>$ MMP-9 expression was higher in animals not reperfused than those fully or partially reperfused, particularly in the striatum $(F(3,52)=11.53 ; p<0.001)$ (Fig 6) and anterior temporal cortex $(F(3,52)=10.35 ; p<0.001)$ (Fig 7$)$.
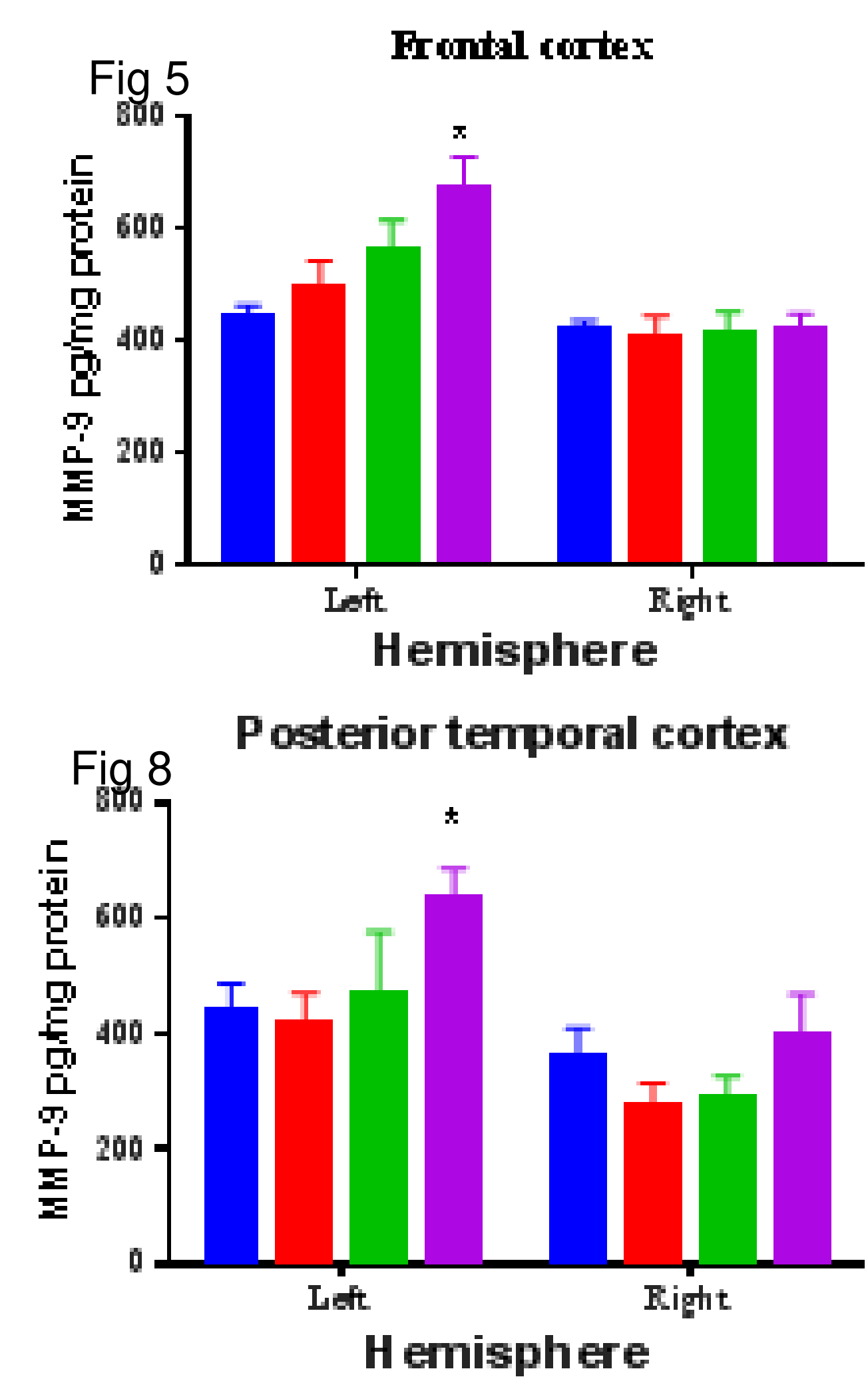

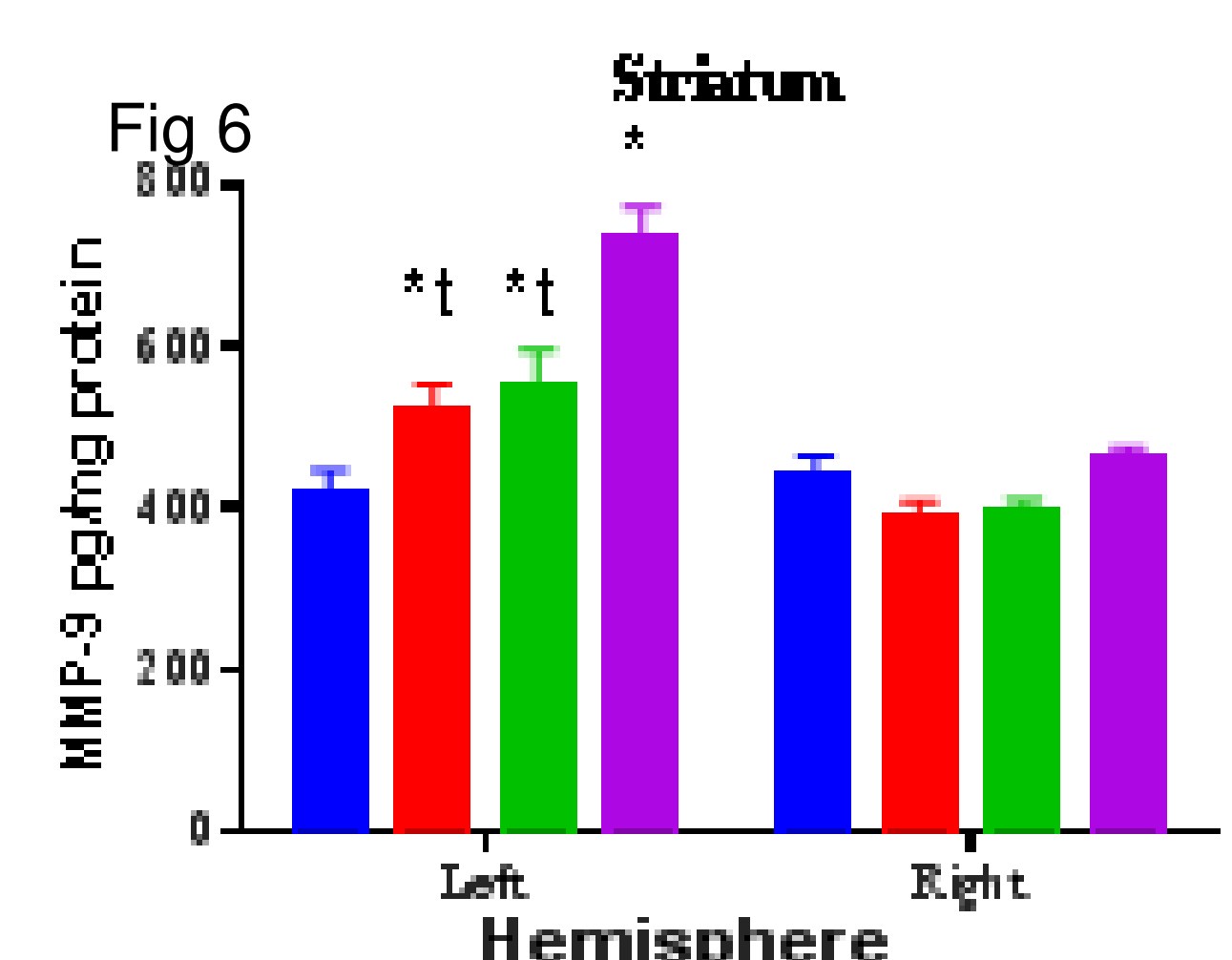

Hipocampe

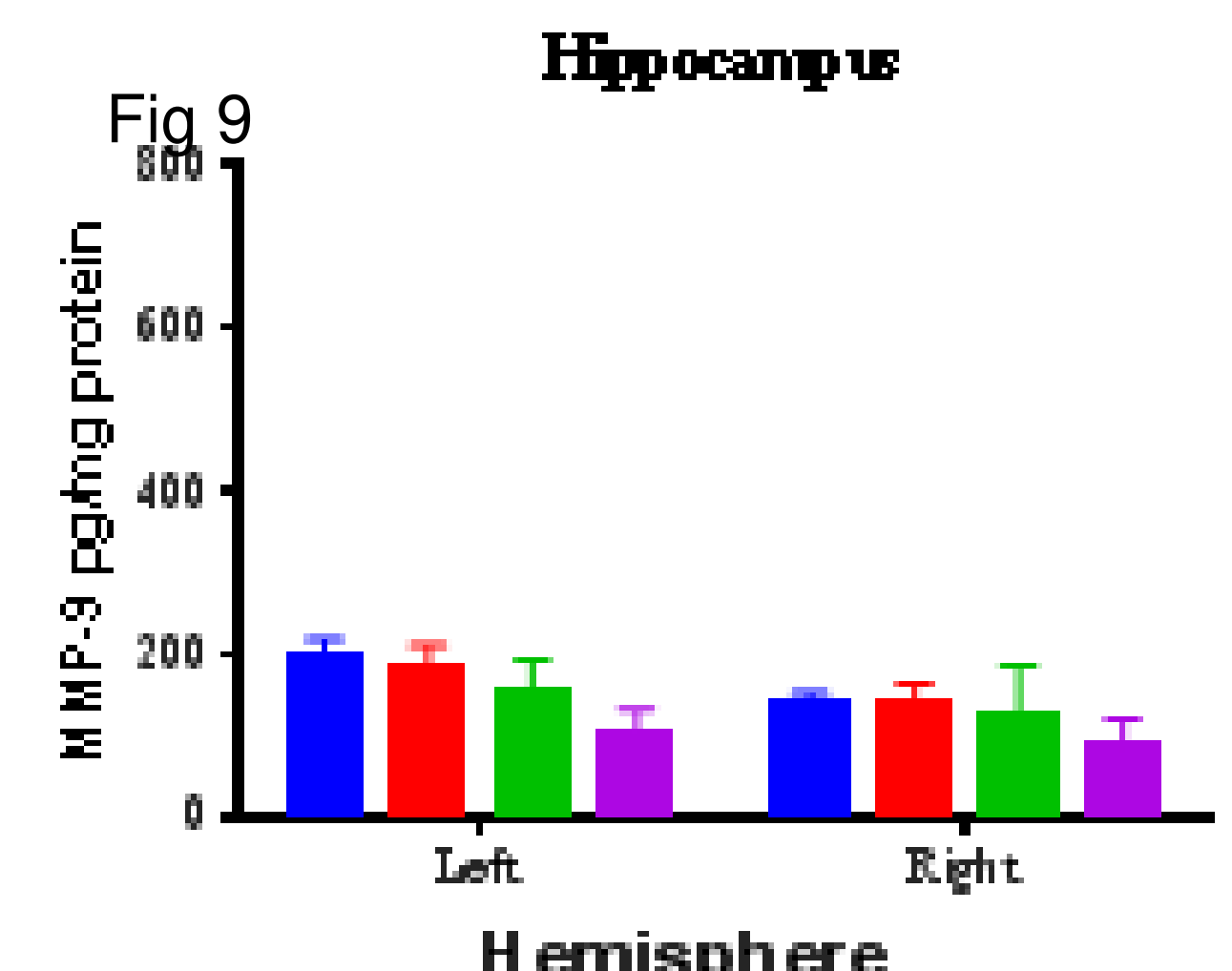

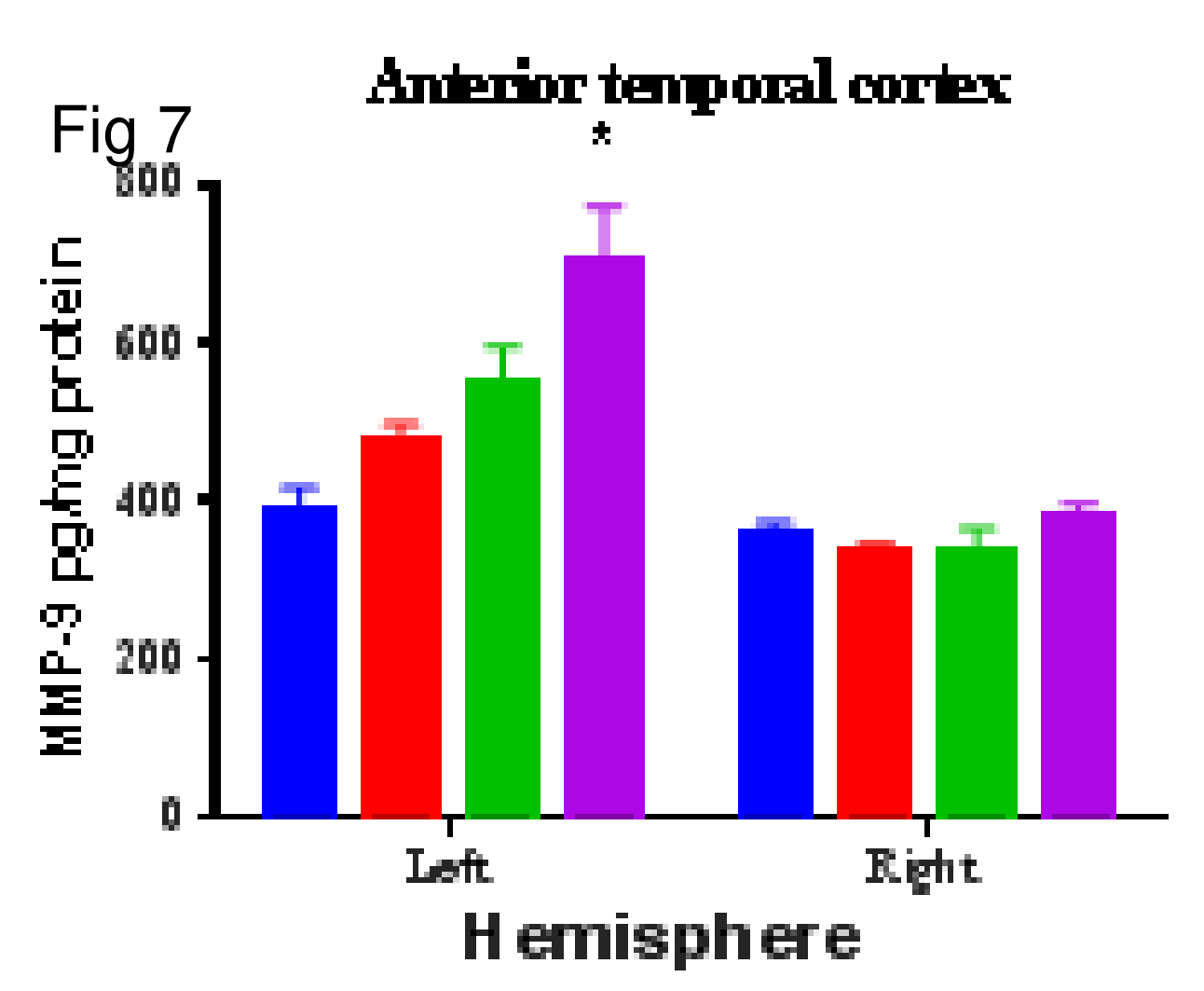

- Sham

Full repertusion
Patial reperiusion

Patial reperisision
Fig 5-9: MMP-9 expression in brain regions in the lesioned and unlesioned hemispheres. Statistical analysis by 2-way ANOVA with Tukey's multiple comparison test $* p<0.05$ vs ipsilateral sham; $t p<0.05$ vs ipsilateral no reperfusion

\section{CONCLUSION}

- Permanent occlusion resulted in greater infarct, oedema and MMP-9 expression than full or partial reperfusion, supporting the clinical benefits of early thrombectomy and indicating the benefit of even partial reperfusion.

\section{ACKNOWLEDGEMENTS}

The authors would like to acknowledge Enterprise Ireland, EU and Ceronovus for financial support. Grant EI IP/2014/0272
ENTERPRISE IRELAND CERENOVUS 